\title{
Establishment of Approximate Analytical Model of Oil Film Force for Finite Length Tilting Pad Journal Bearings
}

\author{
Yongliang Wang, ${ }^{1}$ Yu Gao, ${ }^{1}$ Ying Cui, ${ }^{1}$ and Zhansheng $\mathrm{Liu}^{2}$ \\ ${ }^{1}$ Marine Engineering College, Dalian Maritime University, Dalian, Liaoning 116026, China \\ ${ }^{2}$ School of Energy Science and Engineering, Harbin Institute of Technology, Harbin, Heilongjiang 150001, China \\ Correspondence should be addressed to Yongliang Wang; vibw@163.com
}

Received 26 January 2015; Accepted 29 April 2015

Academic Editor: Paolo Pennacchi

Copyright (c) 2015 Yongliang Wang et al. This is an open access article distributed under the Creative Commons Attribution License, which permits unrestricted use, distribution, and reproduction in any medium, provided the original work is properly cited.

\begin{abstract}
Tilting pad bearings offer unique dynamic stability enabling successful deployment of high-speed rotating machinery. The model of dynamic stiffness, damping, and added mass coefficients is often used for rotordynamic analyses, and this method does not suffice to describe the dynamic behaviour due to the nonlinear effects of oil film force under larger shaft vibration or vertical rotor conditions. The objective of this paper is to present a nonlinear oil force model for finite length tilting pad journal bearings. An approximate analytic oil film force model was established by analysing the dynamic characteristic of oil film of a single pad journal bearing using variable separation method under the dynamic $\pi$ oil film boundary condition. And an oil film force model of a four-tilting-pad journal bearing was established by using the pad assembly technique and considering pad tilting angle. The validity of the model established was proved by analyzing the distribution of oil film pressure and the locus of journal centre for tilting pad journal bearings and by comparing the model established in this paper with the model established using finite difference method.
\end{abstract}

\section{Introduction}

Tilting pad journal bearings are widely used in large steam turbine-generator, heavy duty gas turbines, vertically oriented machines, and so forth, due to their excellent dynamic stability compared with other oil film bearing designs [1-3]. Dynamic performance of tilting pad bearing is one of the important parameters affecting the dynamic characteristics of high-speed rotor supported by tilting pad journal bearings.

In system with horizontal rotors, when the journal dynamic displacements about the equilibrium position are far smaller than the bearing clearance, the bearing reaction forces can be expressed as a stiffness, damping, and added mass coefficients model $[4,5]$, but lager amplitude journal motions mean the linear model has limits to apply. And in vertical rotors with tilting pad bearing, there is no equilibrium position; for example, in vertical hydropower units and pumps, the position of rotor determined by the generator's properties, the flow conditions in the turbine, the mass unbalance, and the linear oil film force models cannot perform simulations of a vertical rotor's behavior
[2]. It is therefore of great significance to calculate the nonlinear oil film force of a tilting pad journal bearing for rotordynamic analysis under severe unbalance conditions or large amplitude journal motions [6].

"Lund's pad assembly method" presented by Lund in 1964 is a commonly known procedure for determining the dynamic characteristics of tilting pad journal bearing [7]; many design and analysis works of tilting pad journal bearing have been done in the last 60 years based on "Lund's pad assembly method" $[8,9]$. And the nonlinear oil film force of a tilting pad bearing can be calculated at present using numerical method $[10,11]$, data base method $[12,13]$, or nonlinear oil film force formula, and so forth. The nonlinear formula is more efficient and convenient compared with the other methods. Okabe and Cavalca $[14,15]$ presented an analytical model of a tilting pad journal bearing with the short bearing assumption and reported that the analytical model was faster than numerical method. The short bearing model has limits in performing dynamic characteristics of tilting pad journal bearing for length-diameter ratio larger than 0.5 . 
The aim of the present work and its main contribution is related to the establishment of an analytical oil film force model of finite length tilting pad journal bearings. An approximate analytic model of a single pad is established through variable separation method under the dynamic $\pi$ oil film boundary condition. And an approximate analytical model of a 4-pad finite length tilting pad journal bearings is presented by using the pad assembly method and by considering pad tilting angle.

\section{Equation for Pad Tilting Angle}

During the calculation of oil film force for tilting pad bearing under given disturbance displacements and velocities of journal, if tilting angle can be quickly obtained, the distribution of oil film thickness can be obtained, and consequently the dynamic characteristics of oil film force of the tilting pad are obtained efficiently.

As shown in Figure 1(a), a pad is installed on supporting block, the journal is directly above the pad, and the gap between the pad and the journal is full of lubricant. Assume that the journal is motionless at the beginning and that $O$ as the center of pad arc, $O_{j}$ as the center of journal, and $\mathrm{O}_{p}$ as the pivot of pad are on the same straight line. The distance between the center of journal and the center of pad, $\left|O O_{j}\right|$, is defined as original eccentricity $e_{0}$, and the original eccentricity ratio is defined as $\varepsilon_{0}=e_{0} / C$, where $C$ is bearing radius clearance.

When the journal starts to rotate clockwise at angular velocity $\Omega$, assume $O_{j}$ is motionless while the journal is rotating. According to fluid lubrication theory, an oil film is formed and pressure is generated under the shear stress of fluid film, as the lubricant is moved between the journal and the pad. After the pad and the neck journal get stable, the tilting pad will rotate through angle $\varphi$ about the pivot point. As shown in Figure 1(b), angle $\varphi$ is the pad tilting angle.

The relationship among pad tilting angle $\varphi$, original eccentricity $\varepsilon_{0}$, and tilting bearings clearance ratio $\psi$, with a wrap angle of $80^{\circ}$ and a pad pivot offset of $50 \%$, can be expressed as shown below as reported in [16]:

$$
\varphi=\varphi\left(\varepsilon_{0}, \psi\right)=\frac{\psi \cdot \varepsilon_{0} \cdot\left(1-\varepsilon_{0}\right) \cdot a_{1}}{\varepsilon_{0}^{5}+\sum_{i=0}^{4}\left(a_{i+2} \cdot \varepsilon_{0}^{i}\right)},
$$

where $a_{1}=0.1172, a_{2}=0.02246, a_{3}=0.8491, a_{4}=-3.069$, $a_{5}=4.807$, and $a_{6}=-3.598$.

\section{Analysis of Oil Film Characteristics of a Single Tilting Pad}

3.1. Oil Film Force Model for Cylindrical Journal Bearing. Figure 2 illustrates the structure of oil film in cylindrical journal bearing, where $R$ is the radius, $O$ is bearing center, and $O_{j}$ is journal center. The journal rotates at velocity $\Omega$ in the bearing and $E$ is the eccentricity of journal in bearing. The

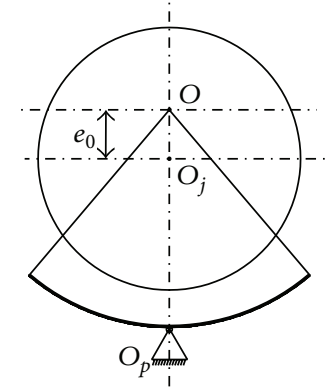

(a)

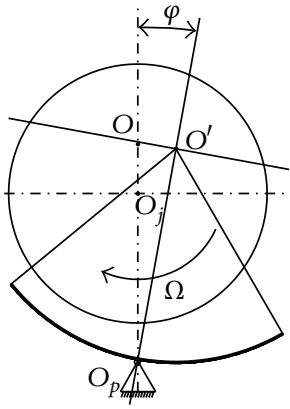

(b)
FIGURE 1: Schematic diagram of pad-swing angle. (a) With journal motionless. (b) With journal rotating at velocity $\Omega$.

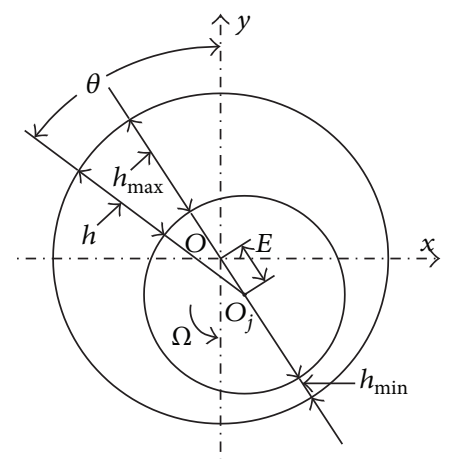

FIGURE 2: Structure of oil film in cylindrical journal bearing.

$x \mathrm{O} y$ Cartesian coordinate is established as shown in Figure 2. The journal displacement and velocity are setting as $x, y, \dot{x}$, and $\dot{y}$, respectively. $\theta$ is circumferential coordinate, while the start is $y$-axis positive and the direction is anticlockwise and $h$ is the oil film thickness.

According to fluid lubrication theory, the nondimensional Reynolds equation of oil film pressure $p$ in a cylindrical journal bearings can be expressed as

$$
\begin{aligned}
& \frac{\partial}{\partial \theta}\left(H^{3} \frac{\partial P}{\partial \theta}\right)+\frac{\partial}{\partial \zeta}\left(H^{3} \frac{\partial P}{\partial \zeta}\right) \\
& \quad=(Y+2 \dot{X}) \sin \theta+(X-2 \dot{Y}) \cos \theta
\end{aligned}
$$

The corresponding nondimensional boundary conditions can be expressed as

$$
\begin{gathered}
P\left(\theta_{1}, \zeta\right)=P\left(\theta_{2}, \zeta\right)=0, \\
P(\theta,-\lambda)=P(\theta, \lambda)=0,
\end{gathered}
$$

where $P=p /\left\{6 \mu \Omega(R / C)^{2}\right\}$ is the nondimensional oil film pressure; $C$ is radius clearance of bearing; $\mu$ is the viscosity of lubrication oil; $D$ is the diameter of bearing; $L$ is the length of bearing; $\lambda=L / D$ is the length-diameter ratio; $z$ is the axial coordinate; $\zeta=z / R$ is the nondimensional lengthdiameter ratio; and $X=x / C, Y=y / C, \dot{X}=\dot{x} /(C \Omega)$, and $\dot{Y}=\dot{y} /(C \Omega)$ are nondimensional displacements and 
velocities of disturbance, respectively. $H=1-Y \cos \theta+X \sin \theta$ is the thickness of the nondimensional oil film; $\tau=t \Omega$ is the nondimensional time; and $t$ is the time.

The inlet and outlet circumferential boundary conditions for oil film under the dynamic $\pi$ boundary conditions can be expressed as shown below:

$$
\begin{aligned}
\theta_{1}= & \arctan \frac{2 \dot{Y}-X}{Y+2 \dot{X}}-\frac{\pi}{2} \operatorname{sign}\left(\frac{2 \dot{Y}-X}{Y+2 \dot{X}}\right) \\
& +\frac{\pi}{2} \operatorname{sign}(2 \dot{Y}-X)+\pi, \\
\theta_{2}= & \theta_{1}+\pi .
\end{aligned}
$$

The approximate analytic model of nondimensional oil film pressure can be established as shown below using variable separation method under the dynamic $\pi$ boundary conditions as reported in [17]:

$$
\begin{aligned}
& P(\theta, \zeta)=\frac{\left(\mathrm{e}^{a \zeta}+\mathrm{e}^{-a \zeta}-\mathrm{e}^{a \lambda}-\mathrm{e}^{-a \lambda}\right)}{\left(\mathrm{e}^{a \lambda}+\mathrm{e}^{-a \lambda}\right)}\left\{C_{1} I_{3}(\theta)\right. \\
& \left.\quad+(Y+2 \dot{X}) I_{1}(\theta)-(X-2 \dot{Y}) I_{2}(\theta)+C_{2}\right\},
\end{aligned}
$$

where

$$
\begin{aligned}
& C_{1}=-(Y+2 \dot{X})\left\{\frac{I_{1}}{I_{3}}\right\}+(X-2 \dot{Y})\left\{\frac{I_{2}}{I_{3}}\right\}, \\
& C_{2}=(Y+2 \dot{X})\left\{\frac{I_{1}\left(\theta_{2}\right) I_{3}\left(\theta_{1}\right)-I_{1}\left(\theta_{1}\right) I_{3}\left(\theta_{2}\right)}{I_{3}}\right\}-(X-2 \dot{Y})\left\{\frac{I_{2}\left(\theta_{2}\right) I_{3}\left(\theta_{1}\right)-I_{2}\left(\theta_{1}\right) I_{3}\left(\theta_{2}\right)}{I_{3}}\right\},
\end{aligned}
$$

$$
\begin{aligned}
& =\sqrt{\frac{\cos \theta_{1}\left\{(Y+2 \dot{X}) I_{5}+(X-2 \dot{Y}) I_{6}\right\}-\sin \theta_{1}\left\{(Y+2 \dot{X}) I_{6}+(X-2 \dot{Y}) I_{4}\right\}}{\left\{\cos \theta_{1}\left[(Y+2 \dot{X})\left(I_{4}-\left(I_{1} / I_{3}\right) I_{1}\right)-(X-2 \dot{Y})\left(I_{6}-\left(I_{2} / I_{3}\right) I_{1}\right)\right]+\sin \theta_{1}\left[(Y+2 \dot{X})\left(I_{6}-\left(I_{1} / I_{3}\right) I_{2}\right)-(X-2 \dot{Y})\left(I_{5}-\left(I_{2} / I_{3}\right) I_{2}\right)\right]\right\}}} \\
& I_{1}(\theta)=\int \frac{\cos \theta}{H^{3}} d \theta \\
& I_{2}(\theta)=\int \frac{\sin \theta}{H^{3}} d \theta ; \\
& I_{3}(\theta)=\int \frac{1}{H^{3}} d \theta ; \\
& I_{4}(\theta)=\int \frac{\cos ^{2} \theta}{H^{3}} d \theta ; \\
& I_{5}(\theta)=\int \frac{\sin ^{2} \theta}{H^{3}} d \theta ; \\
& I_{6}(\theta)=\int \frac{\sin ^{2} \cos \theta}{H^{3}} d \theta .
\end{aligned}
$$

Consider $I_{i}=I_{i}\left(\theta_{1}\right)-I_{i}\left(\theta_{2}\right), i=1,2,3,4,5,6$. The expression of $I_{i}(\theta)$ is shown in the Appendix.

The expression of oil film force can be established by integrating the oil film force along the oil film domain as shown below:

$$
\begin{aligned}
\left\{\begin{array}{l}
F_{x} \\
F_{y}
\end{array}\right\}= & \frac{6 \mu \Omega R^{4}}{C^{2}}\left\{\begin{array}{l}
f_{x} \\
f_{y}
\end{array}\right\} \\
= & \frac{12 \mu \Omega R^{4}}{C^{2} I_{3}}\left\{\frac{\mathrm{e}^{a \lambda}-\mathrm{e}^{-a \lambda}}{a\left(\mathrm{e}^{a \lambda}+\mathrm{e}^{-a \lambda}\right)}-\lambda\right\} \\
& \times\left[\begin{array}{cc}
I_{4} I_{3}-I_{1} I_{1} & -I_{6} I_{3}+I_{2} I_{1} \\
-I_{6} I_{3}+I_{1} I_{2} & I_{5} I_{3}-I_{2} I_{2}
\end{array}\right]\left[\begin{array}{l}
(Y+2 \dot{X}) \\
(X-2 \dot{Y})
\end{array}\right] .
\end{aligned}
$$

3.2. Model of Single Tilting Pad Oil Film Force in a Local Coordinate System. As shown in Figure 3, the disturbance displacement and velocity of a neck journal in a local coordinate system are $\left(\eta^{i}, \xi^{i}\right)$ and $\left(\dot{\eta}^{i}, \dot{\xi}^{i}\right)$.

The expression of oil film pressure in a local coordinate system can be established by analyzing the oil film characteristics of a tilting pad in a local coordinate system and by determining the circumferential boundary conditions according to the pad parameters and the dynamic $\pi$ oil film boundary condition for a cylindrical bearing. The dynamic $\pi$ oil film boundary condition in a local coordinate system can be expressed as $\left[\theta_{1}^{\prime}, \theta_{1}^{\prime}+\pi\right]$ and from formula (4) 


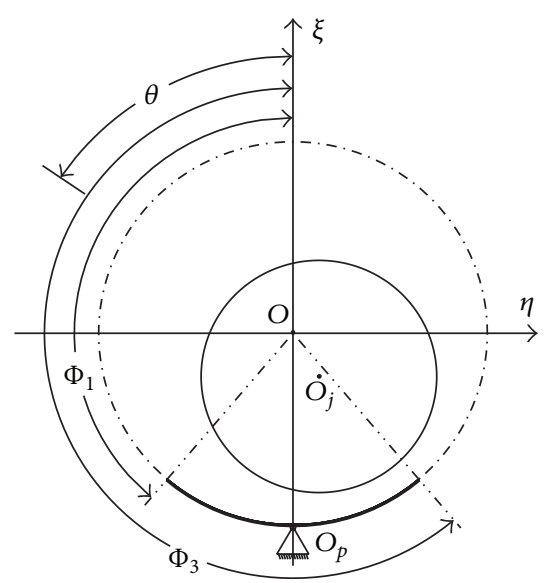

FIgURE 3: Tilting pad oil film in local coordinates system.

$$
\begin{aligned}
\theta_{1}^{\prime}= & \arctan \frac{2\left(\dot{\xi}^{i} / C \Omega\right)-\left(\eta^{i} / C\right)}{\left(\xi^{i} / C\right)+2\left(\dot{\eta}^{i} / C \Omega\right)} \\
& -\frac{\pi}{2} \operatorname{sign}\left(\frac{2\left(\dot{\xi}^{i} / C \Omega\right)-\left(\eta^{i} / C\right)}{\left(\xi^{i} / C\right)+2\left(\dot{\eta}^{i} / C \Omega\right)}\right) \\
& +\frac{\pi}{2} \operatorname{sign}\left(2 \frac{\dot{\xi}^{i}}{C \Omega}-\frac{\eta^{i}}{C}\right)+\pi
\end{aligned}
$$

For a tilting pad, the actual oil film is the area $\left[\theta_{1}^{\prime}, \theta_{1}^{\prime}+\pi\right]$ projected on the pad. The tilting pad bearing can be divided into five areas in the circumferential direction according to its geometric structure. When $\theta_{1}^{\prime}$ is in these areas, the expressions of oil film boundaries for both upper and lower pads are as follows:

$$
\begin{aligned}
& \text { If } 0 \leq \theta_{1}^{\prime}<\Phi_{3}-\pi, \theta_{1}=\Phi_{1}, \theta_{2}=\theta_{1}^{\prime}+\pi \\
& \text { if } \Phi_{3}-\pi \leq \theta_{1}^{\prime}<\Phi_{1}, \theta_{1}=\Phi_{1}, \theta_{2}=\Phi_{3} ; \\
& \text { if } \Phi_{1} \leq \theta_{1}^{\prime}<\Phi_{3}, \theta_{1}=\theta_{1}^{\prime}, \theta_{2}=\Phi_{3} ; \\
& \text { if } \Phi_{3} \leq \theta_{1}^{\prime}<\Phi_{1}+\pi \text {, the oil film pressure is zero; } \\
& \text { if } \Phi_{1}+\pi \leq \theta_{1}^{\prime}<2 \pi, \theta_{1}=\Phi_{1}+2 \pi, \theta_{2}=\theta_{1}^{\prime}+\pi
\end{aligned}
$$

The expression of oil film pressure in a tilting pad under local coordinate is as follows:

$$
\begin{aligned}
& p^{i}(\theta, \zeta)=\frac{\left(\mathrm{e}^{a \zeta}+\mathrm{e}^{-a \zeta}-\mathrm{e}^{a \lambda}-\mathrm{e}^{-a \lambda}\right)}{\left(\mathrm{e}^{a \lambda}+\mathrm{e}^{-a \lambda}\right)}\left\{C_{1} I_{3}(\theta)\right. \\
& +\left(\frac{\xi^{i}}{C}+2 \frac{\dot{\eta}^{i}}{C \Omega}\right) I_{1}(\theta)-\left(\frac{\eta^{i}}{C}-2 \frac{\dot{\xi}^{i}}{C \Omega}\right) I_{2}(\theta) \\
& \left.+C_{2}\right\} .
\end{aligned}
$$

And the dimensionless oil film force of a tilting pad under local coordinate is as follows:

$$
\begin{aligned}
& \left\{\begin{array}{c}
f_{\eta}^{i} \\
f_{\xi}^{i}
\end{array}\right\} \\
& =\frac{2}{I_{3}}\left\{\frac{\mathrm{e}^{a \lambda}-\mathrm{e}^{-a \lambda}}{a\left(\mathrm{e}^{a \lambda}+\mathrm{e}^{-a \lambda}\right)}-\lambda\right\} \\
& \quad \times\left[\begin{array}{cc}
I_{4} I_{3}-I_{1} I_{1} & -I_{6} I_{3}+I_{2} I_{1} \\
-I_{6} I_{3}+I_{1} I_{2} & I_{5} I_{3}-I_{2} I_{2}
\end{array}\right]\left[\begin{array}{l}
\frac{\xi^{i}}{C}+2 \frac{\dot{\eta}^{i}}{C \Omega} \\
\frac{\eta^{i}}{C}-2 \frac{\dot{\xi}^{i}}{C \Omega}
\end{array}\right] .
\end{aligned}
$$

\section{Relations of Oil Film Force in Different Coordinates Systems}

In Figure 4(a), the center of $x \mathrm{O}_{b} y$ coordinate system is on the center of bearings $\mathrm{O}_{b}, \mathrm{O}_{j}$ is the center of journal, the radius of journal is $R$, the radius clearance between the pad and journal is $C$, and the arc radius of the tilting pad is $R+C$.

As shown in Figure 4(b), the pivot point of the pad is determined by the pivot angle $\beta_{i}$, if $O^{\prime}$ is the center of pad arc, and the preload coefficient of the bearings is $m$, and $\overline{\mathrm{O}_{b} \mathrm{O}^{\prime}}=m C$. If the pad rotates through angle $\delta$, the pad arc center moves from $O^{\prime}$ to $O^{\prime \prime}$; the journal center $O_{j}$ is on the line connecting the pivot point and arc center $O^{\prime \prime}$, as shown in Figure 4(c); $\overline{O_{j} O^{\prime \prime}}$ is the original eccentricity $e_{0}$ mentioned above. When the journal rotates at velocity $\Omega$, the pad will consequentially tilt. As shown in Figure 4(d), the pad tilting angle is $\varphi$ and the pad arc center moves from $O^{\prime \prime}$ to $O$.

By setting the coordinates of $\mathrm{NO}, i$ pad pivot in a global coordinate system as $\left(x_{\mathrm{pad}}^{i}, y_{\mathrm{pad}}^{i}\right)$, their specific expression is as follows:

$$
\begin{aligned}
& x_{\mathrm{pad}}^{i}=-[R+C(1-m)] \sin \beta^{i} ; \\
& y_{\mathrm{pad}}^{i}=[R+C(1-m)] \cos \beta^{i} .
\end{aligned}
$$

Assume dimensionless parameter $\left(X_{\mathrm{pad}}^{i}, Y_{\mathrm{pad}}^{i}\right)$ :

$$
\begin{aligned}
& X_{\mathrm{pad}}^{i}=\frac{x_{\mathrm{pad}}^{i}}{C}=-\left(\frac{1}{\psi}+1-m\right) \sin \beta^{i} \\
& Y_{\mathrm{pad}}^{i}=\frac{y_{\mathrm{pad}}^{i}}{C}\left(\frac{1}{\psi}+1-m\right) \cos \beta^{i} .
\end{aligned}
$$

Angle $\delta$ can be calculated using the triangular relations. As shown in Figure 4(c), according to geometric relationship $\Delta \mathrm{O}_{b} \mathrm{O}_{j} \mathrm{O}_{p}$, 


$$
\begin{aligned}
x^{2}+y^{2} & =[R+C(1-m)]^{2}+\left(x-x_{\mathrm{pad}}^{i}\right)^{2}+\left(y-y_{\mathrm{pad}}^{i}\right)^{2}-2[R+C(1-m)] \sqrt{\left(x-x_{\mathrm{pad}}^{i}\right)^{2}+\left(y-y_{\mathrm{pad}}^{i}\right)^{2}} \cos \left|\delta^{i}\right|, \\
\left|\delta^{i}\right| & =\arccos \left\{\frac{[1 / \psi+1-m]^{2}+\left(X-X_{\mathrm{pad}}^{i}\right)^{2}+\left(Y-Y_{\mathrm{pad}}^{i}\right)^{2}-X^{2}-Y^{2}}{2[1 / \psi+1-m] \sqrt{\left(X-X_{\mathrm{pad}}^{i}\right)^{2}+\left(Y-Y_{\mathrm{pad}}^{i}\right)^{2}}}\right\} .
\end{aligned}
$$

By defining counterclockwise is positive

$$
\delta^{i}= \begin{cases}-\left|\delta^{i}\right| & \text { if }-X \cos \beta^{i}-Y \sin \beta^{i} \leq 0 \\ +\left|\delta^{i}\right| & \text { if }-X \cos \beta^{i}-Y \sin \beta^{i}>0 .\end{cases}
$$

The original eccentricity can be expressed as

$$
\varepsilon_{0}^{i}=\frac{1}{\psi}+1-\sqrt{\left(X-X_{\mathrm{pad}}^{i}\right)^{2}+\left(Y-Y_{\mathrm{pad}}^{i}\right)^{2}} .
$$

After pad original eccentricity $\varepsilon_{0}^{i}$ is obtained, pad tilting angle $\varphi^{i}$ can be calculated using (1) with a pad clearance ratio $\psi$.

The corresponding local coordinate system is defined in Figure 5 which is an enlarged view of Figure 4(d), where coordinate $x \mathrm{O}_{b} y$ is a global coordinate system with its origin at the bearing center $O_{b}$ and $(\vec{x}, \vec{y})$ as its basis; $u O_{b} v$ is a local coordinate system fixed on the pad fulcrum and bearing center $O_{b}$, with $(\vec{u}, \vec{v})$ as its basis; and $\eta O \xi$ is a moving coordinate system with its original at the pad center $O$ and $\left(\vec{\eta}^{i}, \vec{\xi}^{i}\right)$ as its basis.

Using the algorithm for space vector, the following expression can be established in accordance with the geometric relationship shown in Figure 5:

$$
\begin{aligned}
\left(\begin{array}{l}
\eta^{i} \\
\xi^{i}
\end{array}\right)= & P^{i} Q^{i}\left(\begin{array}{l}
x \\
y
\end{array}\right) \\
& +\left(\begin{array}{c}
-\sin \phi^{i}(R+C(1-m)) \\
\cos \phi^{i}(R+C(1-m))-(R+C)
\end{array}\right),
\end{aligned}
$$

where

$$
\begin{array}{r}
Q^{i}=\left[\begin{array}{rr}
-\cos \beta^{i} & -\sin \beta^{i} \\
\sin \beta^{i} & -\cos \beta^{i}
\end{array}\right], \\
P^{i}=\left[\begin{array}{cc}
\cos \phi^{i} & -\sin \phi^{i} \\
\sin \phi^{i} & \cos \phi^{i}
\end{array}\right] \\
\phi^{i}=\delta^{i}-\varphi^{i} .
\end{array}
$$

Similarly, the transitions of velocity are

$$
\left(\begin{array}{c}
\dot{\eta}^{i} \\
\dot{\xi}^{i}
\end{array}\right)=P^{i} Q^{i}\left(\begin{array}{c}
\dot{x} \\
\dot{y}
\end{array}\right) \text {. }
$$

And the relation of dimensionless oil film force under global coordinate system and local coordinate system is as follows:

$$
\left(\begin{array}{c}
F_{x}^{i}(x, y, \dot{x}, \dot{y}) \\
F_{y}^{i}(x, y, \dot{x}, \dot{y})
\end{array}\right)=\left[P^{i} Q^{i}\right]^{-1}\left(\begin{array}{c}
F_{\eta}^{i}\left(\eta^{i}, \xi^{i}, \dot{\eta}^{i}, \dot{\xi}^{i}\right) \\
F_{\xi}^{i}\left(\eta^{i}, \xi^{i}, \dot{\eta}^{i}, \dot{\xi}^{i}\right)
\end{array}\right) .
$$
follows:

The dimensionless forms of (17), (19), and (20) are as

$$
\begin{aligned}
& \left(\begin{array}{c}
\frac{\eta^{i}}{C} \\
\frac{\xi^{i}}{C}
\end{array}\right) \\
& =P^{i} Q^{i}\left(\begin{array}{l}
X \\
X
\end{array}\right) \\
& +\left(\begin{array}{c}
-\sin \phi^{i}\left(\frac{1}{\psi}+1-m\right) \\
\cos \phi^{i}\left(\frac{1}{\psi}+1-m\right)-\left(\frac{1}{\psi}+1\right)
\end{array}\right) \\
& \left(\begin{array}{c}
\frac{\dot{\eta}^{i}}{(C \Omega)} \\
\frac{\dot{\xi}^{i}}{(C \Omega)}
\end{array}\right)=P^{i} Q^{i}\left(\begin{array}{l}
\dot{X} \\
\dot{Y}
\end{array}\right), \\
& \left(\begin{array}{l}
f_{x}^{i}(X, Y, \dot{X}, \dot{Y}) \\
f_{y}^{i}(X, Y, \dot{X}, \dot{Y})
\end{array}\right) \\
& =\left[P^{i} Q^{i}\right]^{-1}\left(\begin{array}{l}
f_{\eta}^{i}\left(\frac{\eta^{i}}{C}, \frac{\xi^{i}}{C}, \frac{\dot{\eta}^{i}}{(C \Omega)}, \frac{\dot{\xi}^{i}}{(C \Omega)}\right. \\
f_{\dot{\xi}}^{i}\left(\frac{\eta^{i}}{C}, \frac{\xi^{i}}{C}, \frac{\dot{\eta}^{i}}{(C \Omega)}, \frac{\dot{\xi}^{i}}{(C \Omega)}\right)
\end{array}\right) .
\end{aligned}
$$

When disturbance displacement $(x, y)$ and velocity $(\dot{x}, \dot{y})$ of a journal in bearings are known, disturbance displacement $\left(\eta^{i}, \xi^{i}\right)$ and velocity $\left(\dot{\eta}^{i}, \dot{\xi}^{i}\right)$ of the journal in a local coordinate system can be calculated using (17) and (19). Nondimensional oil film force $\left(f_{\eta}^{i}, f_{\xi}^{i}\right)$ is calculated in the local coordinate system of the pad. Finally, it can be transformed to using (23) into the oil film force $\left(f_{x}^{i}, f_{y}^{i}\right)$ in a global coordinates system. 


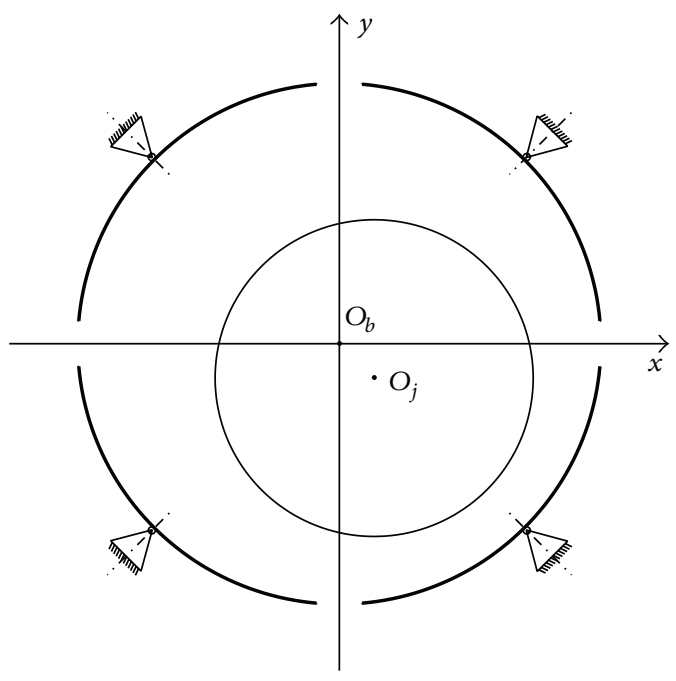

(a)

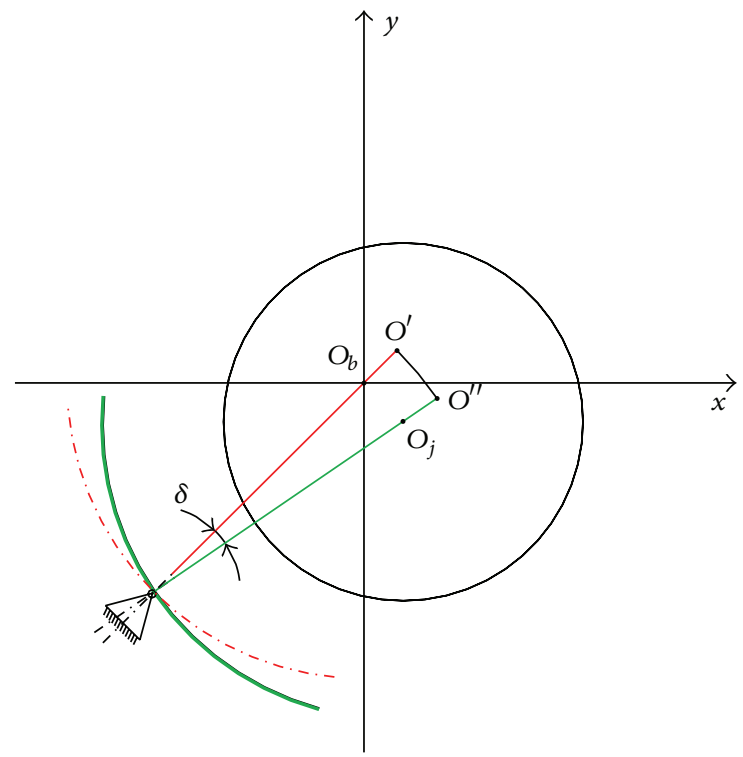

(c)

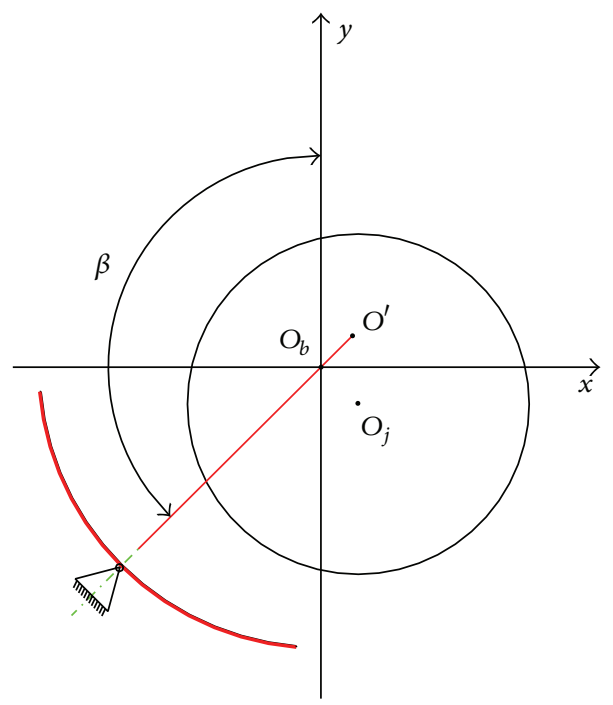

(b)

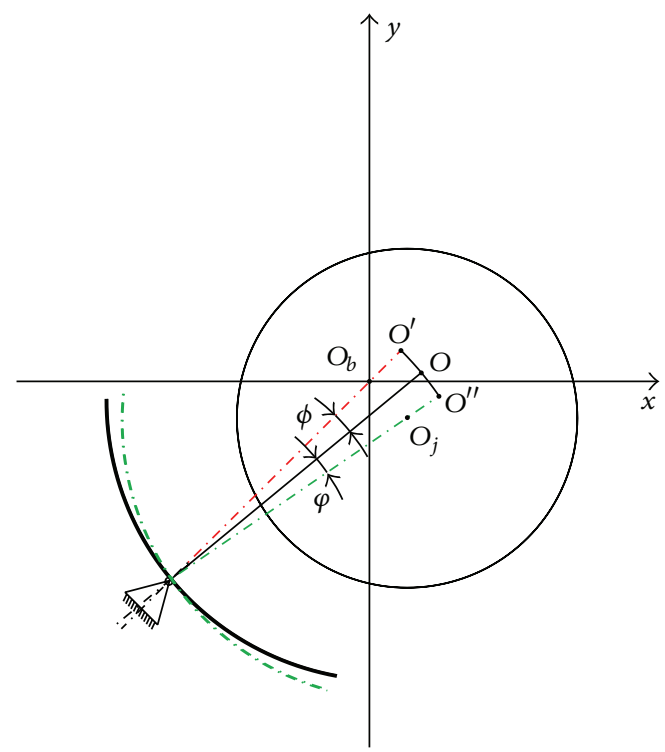

(d)

FIgURE 4: Tilting pad bearing and rotor journal.

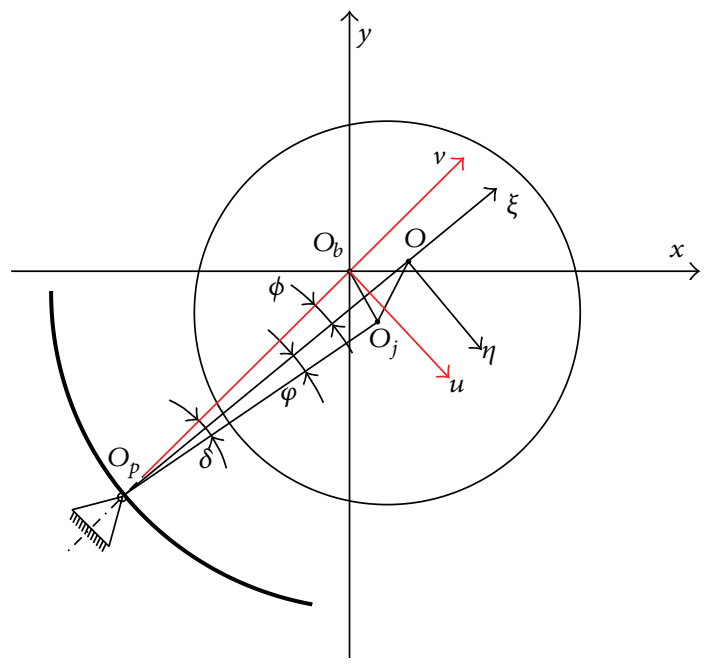

FIGURE 5: Relationship of coordinates systems. 


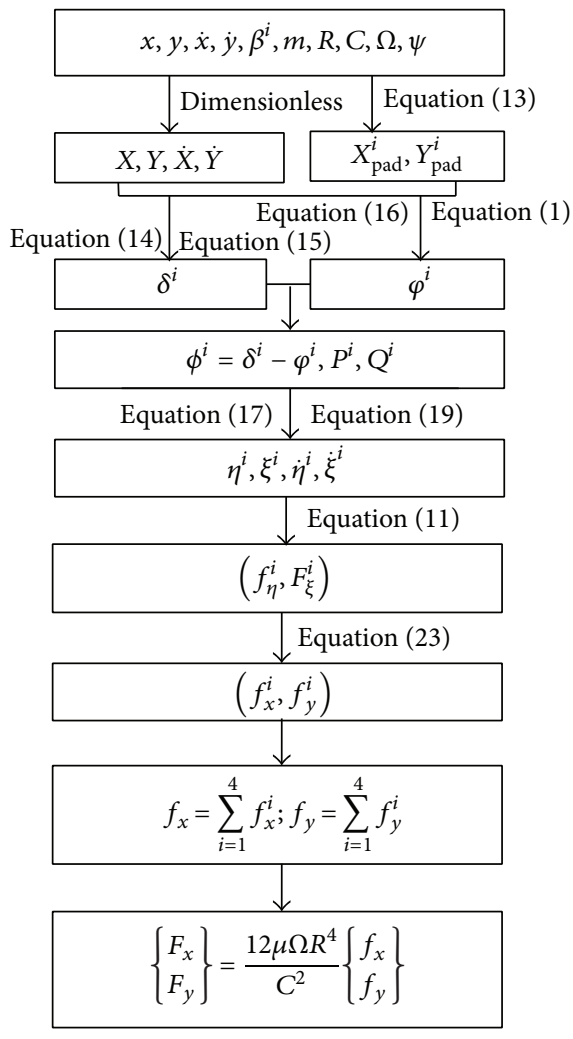

FIGURE 6: Process of solving oil film force of tilting pad bearing.

\section{Approximate Analysis Model of Oil Film Force for Tilting Pad Bearing}

The nonlinear oil film force of a four-tilting-pad bearing can be calculated through the process shown in Figure 6 when the parameters of bearing, disturbance displacement, and disturbance velocity of a journal in the bearing are identified.

\section{Analysis of Oil Film Characteristics of Tilting Pad Bearing}

The dimensionless pressure distributions of tilting pad journal bearing are got by evaluating (10) for different lengthdiameter ratio $(L / D=0.1,0.5,1,5)$, shown in Figure 7, when $E=0.1(X=0.1 \cos (\pi / 4), Y=0.1 \sin (\pi / 4)), \dot{X}=0, \dot{Y}=0$, attitude angle of $45^{\circ}, m=0.5$, and $\Psi=3 \%$.

With these parameters, the axis center lies in first quadrant, and the original eccentricities of the pads in second quadrant and fourth quadrant are the same; the oil film pressure distribution of pads \#1 and \#3 in second and fourth quadrant should agree. The original eccentricity in third quadrant is relatively larger than those in other quadrants, and so the oil film pressure of pad \#2 is relatively smaller. Similarly, the oil film pressure of pad \#4 in the fourth quadrant is the largest.
As shown in Figure 7, the dimensionless pressure of oil film increases as the length-diameter ratio increases. The oil film pressure distribution in axial direction exhibits as parabola form when the length-diameter ratio is smaller; the axial distribution of oil film pressure is generally considered consistent when the length-diameter ration reaches 5.

Figure 8 is the displacement of journal center within bearing clearance for different operating conditions, where, $L / D$ the length-diameter ratio of tilting pad bearing is $0.5 ; m$ the preload coefficient is 0.5 ; and, $\Psi$, the clearance ratio is $3 \mathrm{G}$.

The static equilibrium position curve of a tilting pad journal bearing is approximately a line segment along the direction of bearing center, which is quite different from the balanced semicircle of circular-pad journal bearing in an equilibrium position. The static equilibrium position for each state is closer to $y$-axis, because the pad of a journal bearing tilts as the journal center changes with weak horizontal and vertical couplings. As shown in Figure 8(b), the static balance curve is not exactly a straight line but a line with weak fluctuation, which means the oil film forces in horizontal and vertical directions exhibit a weak coupling relationship.

In order to validate the accuracy of the present oil film force model, comparisons have been made with the finite difference method model, and the boundary conditions are 


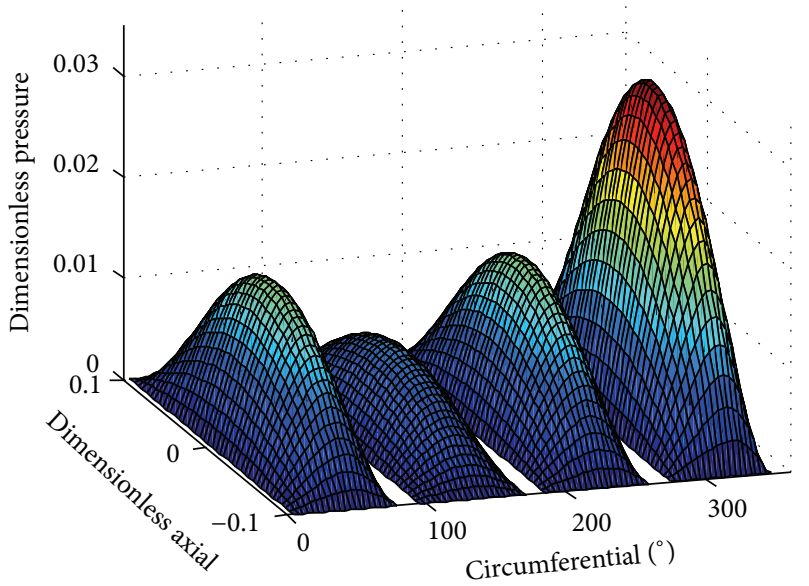

(a)

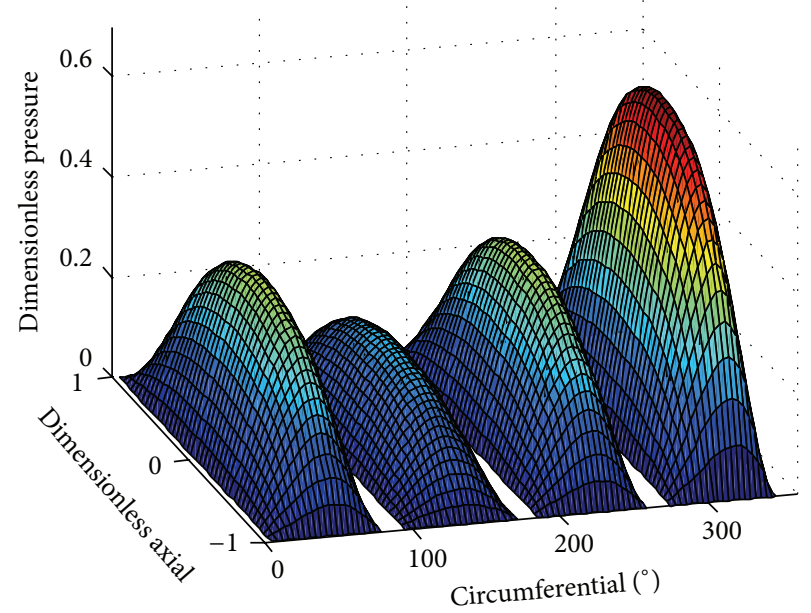

(c)

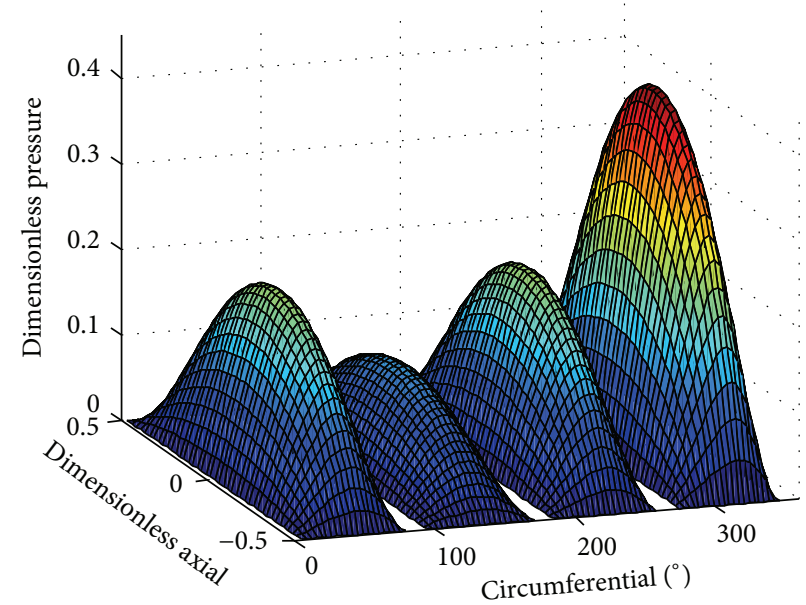

(b)

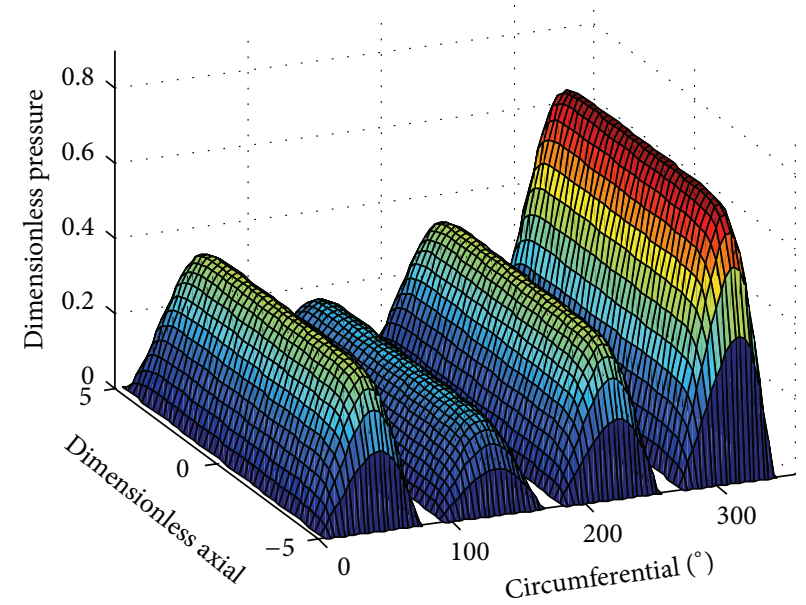

(d)

FIGURE 7: Dimensionless pressure of four-tilting-pad bearing in different $L / D$ : (a) 0.1 , (b) 0.5, (c) 1, and (d) 5 .

consistent in the models; oil film forces for different models with different eccentricities were investigated. Based upon Figure 9, the total oil film force can be derived as follows:

$$
f_{\text {total }}=\sqrt{f_{x}^{2}+f_{y}^{2}} \text {. }
$$

Figure 9 shows the comparison of the total dimensionless oil film force versus original eccentricity from finite difference model with the current approach for different length-diameter ratio, where eccentricity is $0.01-0.49$, the pad preload coefficient $m=0.5$, bearing clearance ratio $\Psi=3 \%$, pad pivot offset is $50 \%$, pad arc angle is $80^{\circ}$, attitude angle is $45^{\circ}$, and length-diameter ratios $L / D=0.01,0.5,1$, and 5.

As shown in Figure 9, the variation of approximate analysis model proposed in the paper is the same as that of the finite difference model for different length-diameter ratios, which means the model proposed in this paper is correct and reliable.

\section{Conclusions}

The oil film characteristics of a single tilting pad are analyzed in this paper, and an oil film force model is established for a single pad under local coordinates, the relations between local and global coordinates are discussed, and a nonlinear oil film force model is proposed for a four-tilting-pad journal bearing.

The model proposed in this paper was proved to be correct and accurate by analyzing the distribution of oil film pressure and by comparing the model proposed in this paper with the model established using finite difference method. 


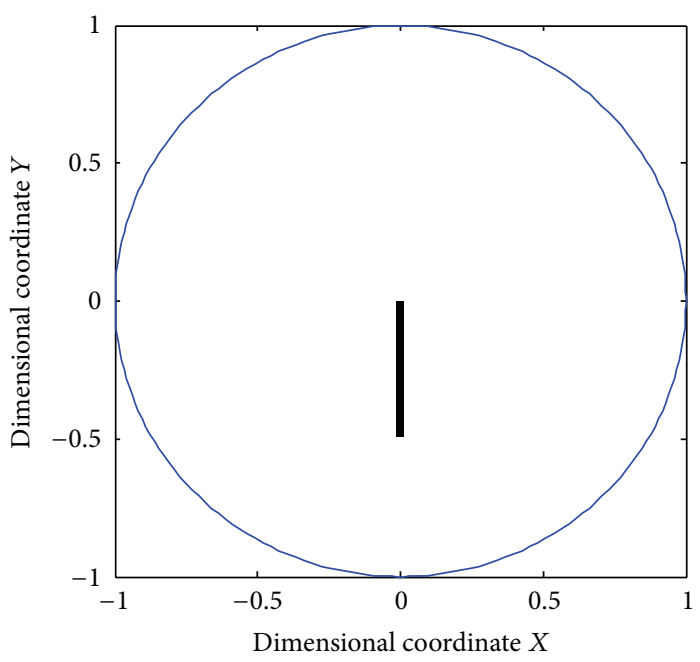

- Static equilibrium position

- Radial clearance circle

(a) Static equilibrium position

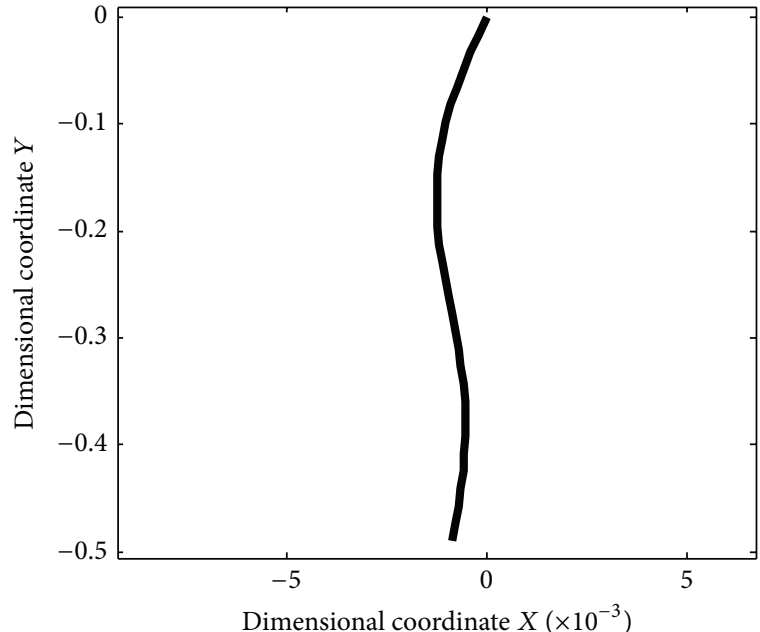

(b) Partial enlarged view

FIGURE 8: Locus of journal center for tilting pad journal bearing.

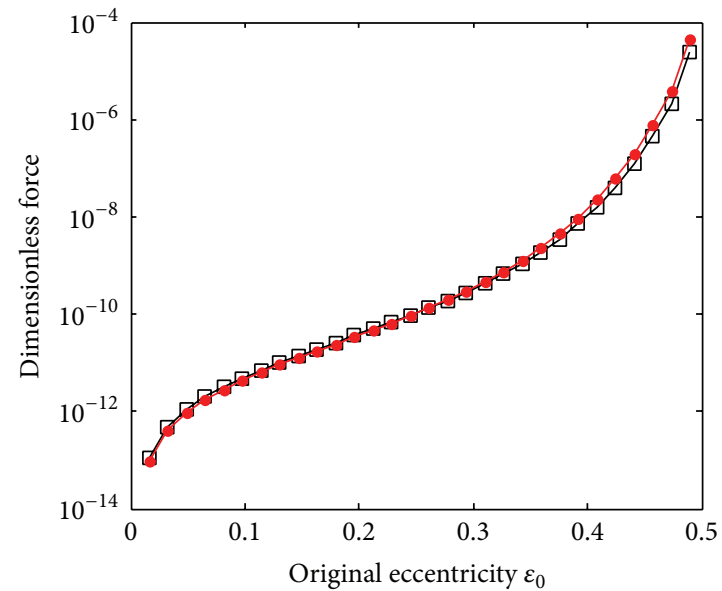

(a) $L / D=0.01$

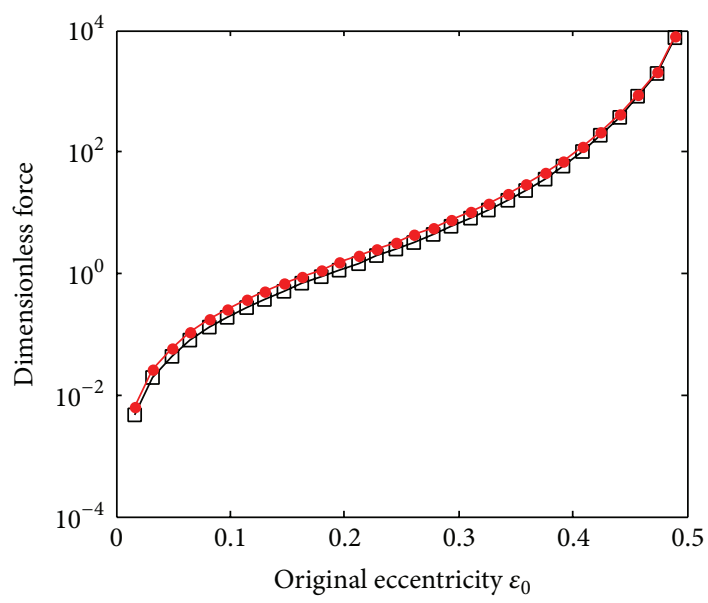

$\square$ The present model

$\rightarrow$ Finite difference model

(c) $L / D=1$

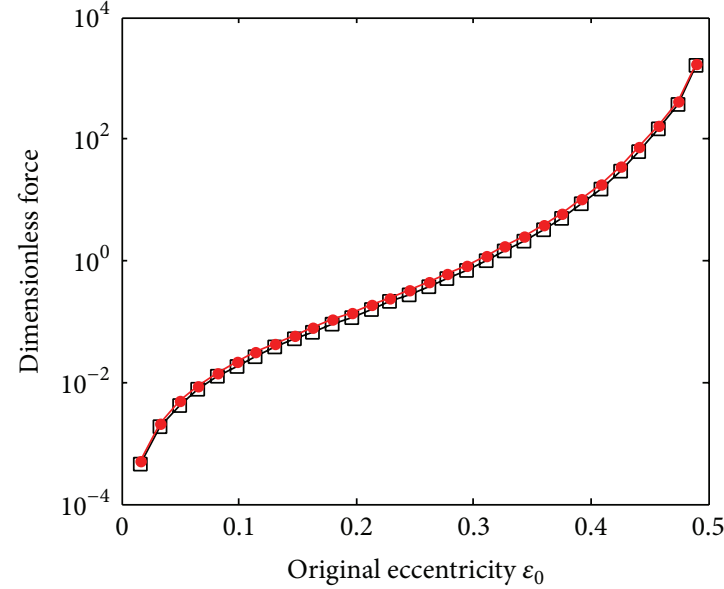

(b) $L / D=0.5$

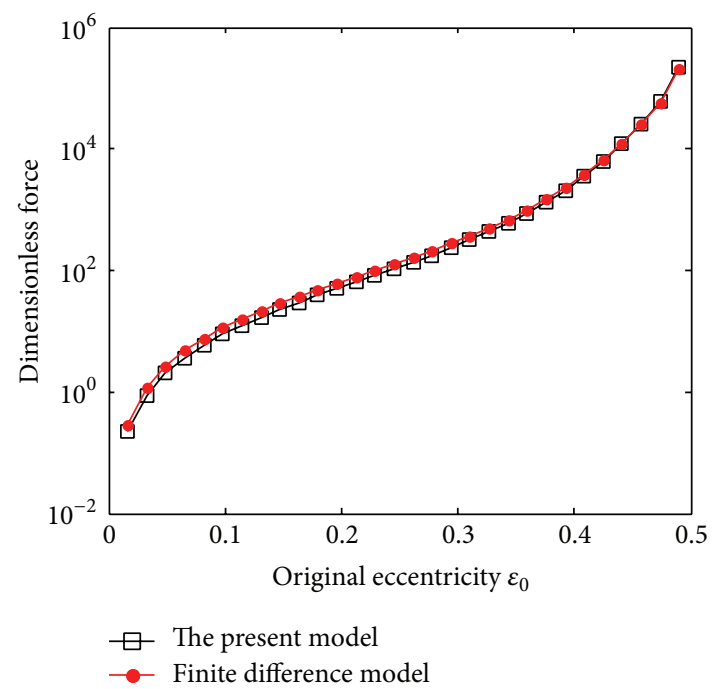

(d) $L / D=5$

FIGURE 9: Dimensionless force versus original eccentricity for different $L / D$ : (a) 0.01 , (b) 0.5 , (c) 1, and (d) 5. 


\section{Appendix}

Consider

$$
\begin{aligned}
& G(X, Y, \theta)= \begin{cases}0, & X=0 \text { and } Y=0 \text { and } \theta=\theta_{1} \\
\frac{\pi}{2}, & X=0 \text { and } Y=0 \text { and } \theta=\theta_{2} \\
\arctan \left(\sqrt{\frac{1-\sqrt{X^{2}+Y^{2}}}{1+\sqrt{X^{2}+Y^{2}}}} \cdot \frac{\sqrt{X^{2}+Y^{2}}+Y \cos \theta-X \sin \theta}{-Y \sin \theta-X \cos \theta}\right), & \text { the other, }\end{cases} \\
& \beta=\theta-\arctan \left(-\frac{X}{Y}\right)+\frac{\pi}{2} \operatorname{sign}\left(-\frac{X}{Y}\right)-\frac{\pi}{2} \operatorname{sign}(-X)-\pi, \\
& I_{1}(\theta)=-\frac{1}{\sqrt{X^{2}+Y^{2}}}\left[Y \int \frac{\cos \beta}{(1+\varepsilon \cos \beta)^{3}} d \beta+X \int \frac{\sin \beta}{(1+\varepsilon \cos \beta)^{3}} d \beta\right] \text {, } \\
& I_{2}(\theta)=\frac{1}{\sqrt{X^{2}+Y^{2}}}\left[-Y \int \frac{\sin \beta}{(1+\varepsilon \cos \beta)^{3}} d \beta+X \int \frac{\cos \beta}{(1+\varepsilon \cos \beta)^{3}} d \beta\right] \text {, } \\
& I_{3}(\theta)=-\frac{1}{2\left(1-X^{2}-Y^{2}\right)} \frac{-Y \sin \theta-X \cos \theta}{1-Y \cos \theta+X \sin \theta}\left(\frac{1}{1-Y \cos \theta+X \sin \theta}+\frac{3}{1-X^{2}-Y^{2}}\right) \\
& +\frac{2+X^{2}+Y^{2}}{\sqrt{\left(1-X^{2}-Y^{2}\right)^{5}}} G(X, Y, \theta) \text {, } \\
& I_{4}(\theta)=\frac{1}{X^{2}+Y^{2}}\left[Y^{2} \int \frac{\cos ^{2} \beta}{(1+\varepsilon \cos \beta)^{3}} d \beta+X^{2} \int \frac{\sin ^{2} \beta}{(1+\varepsilon \cos \beta)^{3}} d \beta+2 X Y \int \frac{\sin \beta \cos \beta}{(1+\varepsilon \cos \beta)^{3}} d \beta\right] \text {, } \\
& I_{5}(\theta)=\frac{1}{X^{2}+Y^{2}}\left[Y^{2} \int \frac{\sin ^{2} \beta}{(1+\varepsilon \cos \beta)^{3}} d \beta+X^{2} \int \frac{\cos ^{2} \beta}{(1+\varepsilon \cos \beta)^{3}} d \beta-2 X Y \int \frac{\sin \beta \cos \beta}{(1+\varepsilon \cos \beta)^{3}} d \beta\right] \text {, } \\
& I_{6}(\theta)=\frac{1}{X^{2}+Y^{2}}\left[\left(Y^{2}-X^{2}\right) \int \frac{\sin \beta \cos \beta}{(1+\varepsilon \cos \beta)^{3}} d \beta-X Y \int \frac{\cos ^{2} \beta}{(1+\varepsilon \cos \beta)^{3}} d \beta+X Y \int \frac{\sin ^{2} \beta}{(1+\varepsilon \cos \beta)^{3}} d \beta\right], \\
& \int \frac{\sin \beta}{(1+\varepsilon \cos \beta)^{3}} d \beta=\frac{1}{2 \sqrt{X^{2}+Y^{2}}(1-Y \cos \theta+X \sin \theta)^{2}} \text {, } \\
& \int \frac{\cos \beta}{(1+\varepsilon \cos \beta)^{3}} d \beta=\frac{1}{2\left(1-X^{2}-Y^{2}\right)} \frac{1}{\sqrt{X^{2}+Y^{2}}} \frac{-Y \sin \theta-X \cos \theta}{1-Y \cos \theta+X \sin \theta}\left(\frac{1}{1-Y \cos \theta+X \sin \theta}+\frac{1+2 X^{2}+2 Y^{2}}{1-X^{2}-Y^{2}}\right) \\
& -\frac{3 \sqrt{X^{2}+Y^{2}}}{\sqrt{\left(1-X^{2}-Y^{2}\right)^{5}}} G(X, Y, \theta), \\
& \int \frac{\cos \beta \sin \beta}{(1+\varepsilon \cos \beta)^{3}} d \beta=\frac{1-2 Y \cos \theta+2 X \sin \theta}{2\left(X^{2}+Y^{2}\right)(1-Y \cos \theta+X \sin \theta)^{2}}, \\
& \int \frac{\sin ^{2} \beta}{(1+\varepsilon \cos \beta)^{3}} d \beta=\frac{-Y \sin \theta-X \cos \theta}{2\left(X^{2}+Y^{2}\right)(1-Y \cos \theta+X \sin \theta)^{2}}-\frac{1}{2\left(X^{2}+Y^{2}\right)\left(1-X^{2}-Y^{2}\right)} \frac{-Y \sin \theta-X \cos \theta}{1-Y \cos \theta+X \sin \theta} \\
& +\frac{1}{\sqrt{\left(1-X^{2}-Y^{2}\right)^{3}}} G(X, Y, \theta) \\
& \int \frac{\cos ^{2} \beta}{(1+\varepsilon \cos \beta)^{3}} d \beta=I_{3}(\theta)-\int \frac{\sin ^{2} \beta}{(1+\varepsilon \cos \beta)^{3}} d \beta \text {. }
\end{aligned}
$$




\section{Conflict of Interests}

The authors declare that there is no conflict of interests regarding the publication of this paper.

\section{Acknowledgment}

This work is funded by the Fundamental Research Funds for the Central Universities (Grant no. 3132015026).

\section{References}

[1] T. Dimond, A. Younan, and P. Allaire, "A review of tilting pad bearing theory," International Journal of Rotating Machinery, vol. 2011, Article ID 908469, 23 pages, 2011.

[2] M. Nässelqvist, R. Gustavsson, and J.-O. Aidanpää, "Experimental and numerical simulation of unbalance response in vertical test rig with tilting-pad bearings," International Journal of Rotating Machinery, vol. 2014, Article ID 309767, 10 pages, 2014.

[3] D. P. Tschoepe and D. W. Childs, "Measurements versus predictions for the static and dynamic characteristics of a four-pad, rocker-pivot, tilting-pad journal bearing," Journal of Engineering for Gas Turbines and Power, vol. 136, no. 5, Article ID 052501, 11 pages, 2014.

[4] L. S. Andrés and Y. J. Tao, "The role of pivot stiffness on the dynamic force coefficients of tilting pad journal bearings," Journal of Engineering for Gas Turbines and Power, vol. 135, no. 11, Article ID 112505, 11 pages, 2013.

[5] D. W. Childs and J. Harris, "Static performance characteristics and rotordynamic coefficients for a four-pad ball-in-socket tilting pad journal bearing," Journal of Engineering for Gas Turbines and Power, vol. 131, no. 6, Article ID 062502, 11 pages, 2009.

[6] J. M. Cao, T. Dimond, and P. Allaire, "Nonlinear modeling of tilting-pad bearings with application to a flexible rotor analysis," in Proceedings of the ASME Design Engineering Technical Conference, Paper no. DETC2013-13712, p. 12, Portland, Ore, USA, August 2013.

[7] J. W. Lund, "Spring and damping coefficients for the tilting-pad journal bearing," ASLE Transactions, vol. 7, pp. 342-352, 1964.

[8] J. C. Nicholas, E. J. Gunter, and P. E. Allaire, "Stiffness and damping coefficients for the five-pad tilting-pad bearing," ASLE Transactions, vol. 22, no. 2, pp. 113-124, 1979.

[9] J. C. Nicholas, "Lund's tilting pad journal bearing pad assembly method," Transactions of the ASME, Journal of Vibration and Acoustics, vol. 125, no. 4, pp. 448-454, 2003.

[10] S. Pagano, E. Rocca, M. Russo, and R. Russo, "Dynamic behaviour of tilting-pad journal bearings," Proceedings of the Institution of Mechanical Engineers J: Journal of Engineering Tribology, vol. 209, no. 4, pp. 275-285, 1995.

[11] R. Brancati, E. Rocca, and R. Russo, "Non-linear stability analysis of a rigid rotor on tilting pad journal bearing," The American Society of Lubrication Engineers, vol. 29, no. 7, pp. 571$578,1996$.

[12] Y. Hou, T. Lai, S. Chen, B. Ma, and J. Liu, "Numerical analysis on the static performance of tilting pad-journal gas bearing in subsystems," Tribology International, vol. 61, pp. 70-79, 2013.

[13] W. Wang and Z. M. Wang, "Nonlinear oil film force database," Journal of Shanghai University of Technology, vol. 14, no. 4, pp. 299-305, 1993.
[14] E. P. Okabe and K. L. Cavalca, "Rotordynamic analysis of systems with a non-linear model of tilting pad bearings," in Proceedings of the 7th International Conference on Rotor Dynamics (IFToMM '06), Paper No. 222, p. 10, Technische Universitat Wien, Vienna, Austria, 2006.

[15] E. P. Okabe and K. L. Cavalca, "Rotordynamic analysis of systems with a non-linear model of tilting pad bearings including turbulence effects," Nonlinear Dynamics, vol. 57, no. 4, pp. 481495, 2009.

[16] Y. L. Wang and Z. S. Liu, "Study on tilting characteristic of pads in tilting pad bearing," in Proceedings of the International Conference on Electronic and Mechanical Engineering and Information Technology (EMEIT '11), vol. 9, pp. 4653-4657, Harbin, China, August 2011.

[17] Y. L. Wang, Z. S. Liu, W. J. Kang, and J. J. Yan, "Approximate analytical model for fluid film force of finite length plain journal bearing," Proceedings of the Institution of Mechanical Engineers Part C: Journal of Mechanical Engineering Science, vol. 226, no. 5, pp. 1345-1355, 2012. 

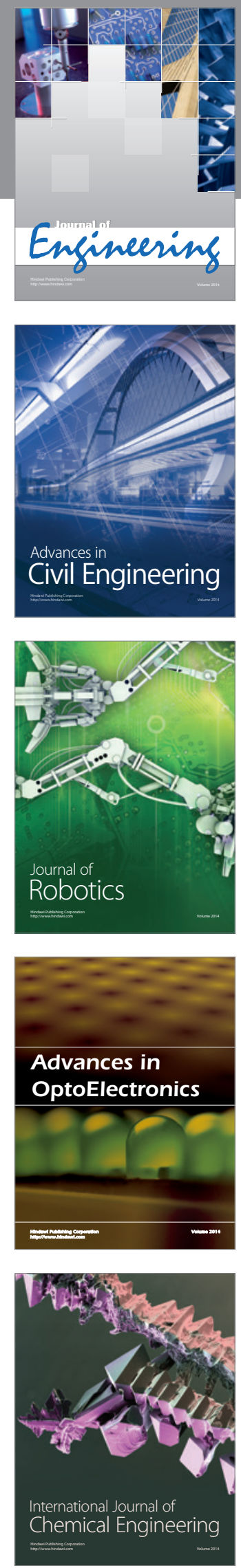

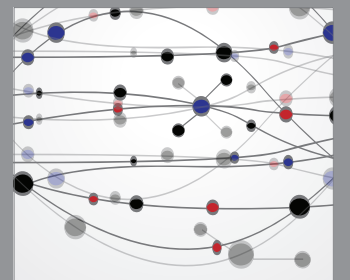

The Scientific World Journal
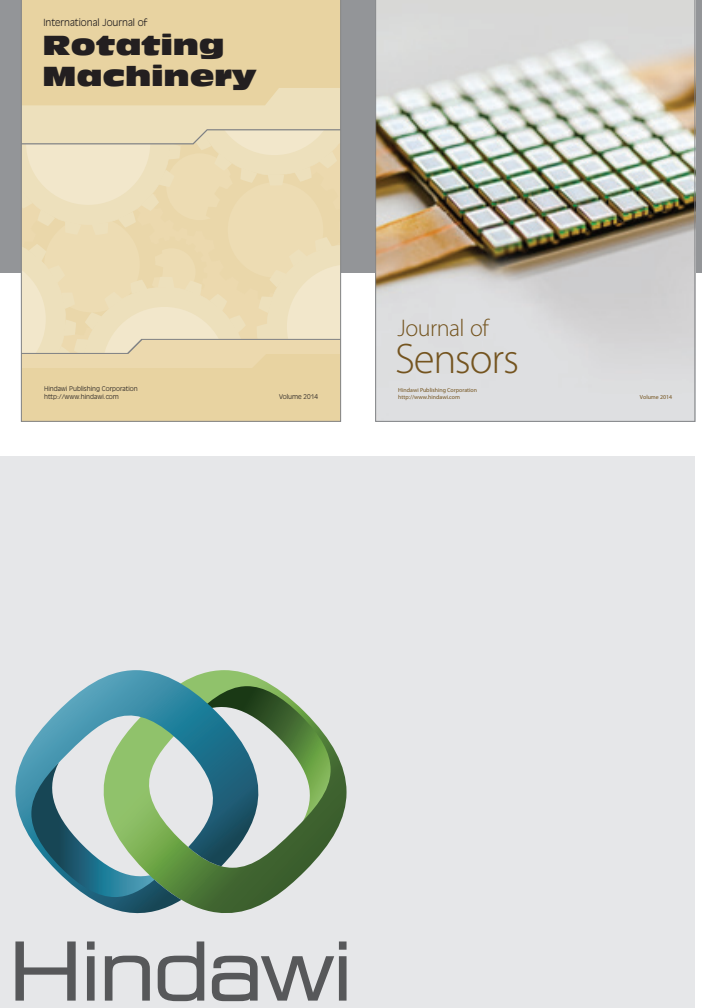

Submit your manuscripts at http://www.hindawi.com
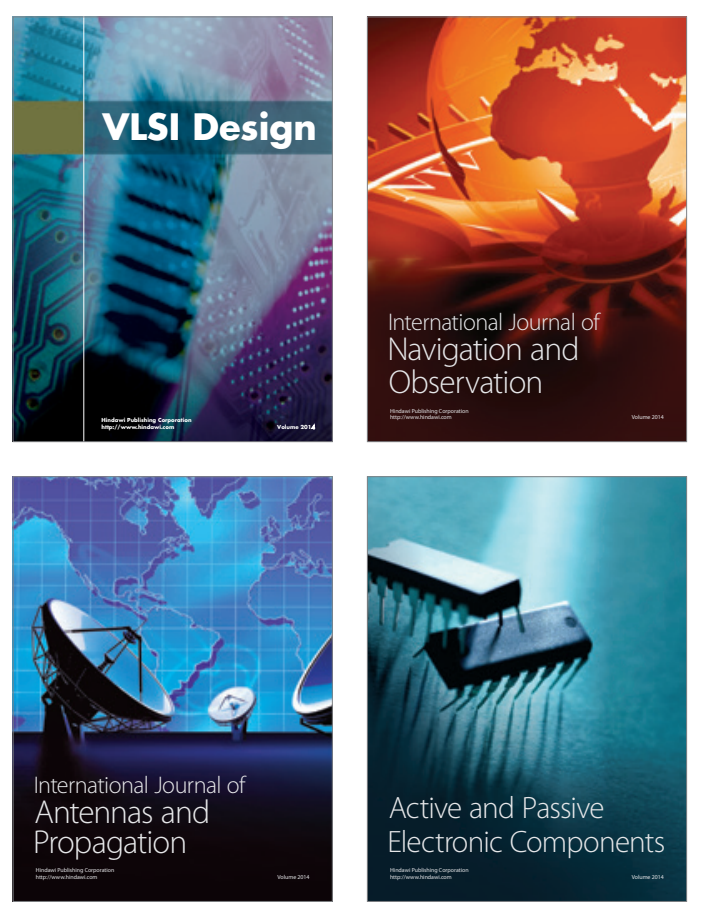
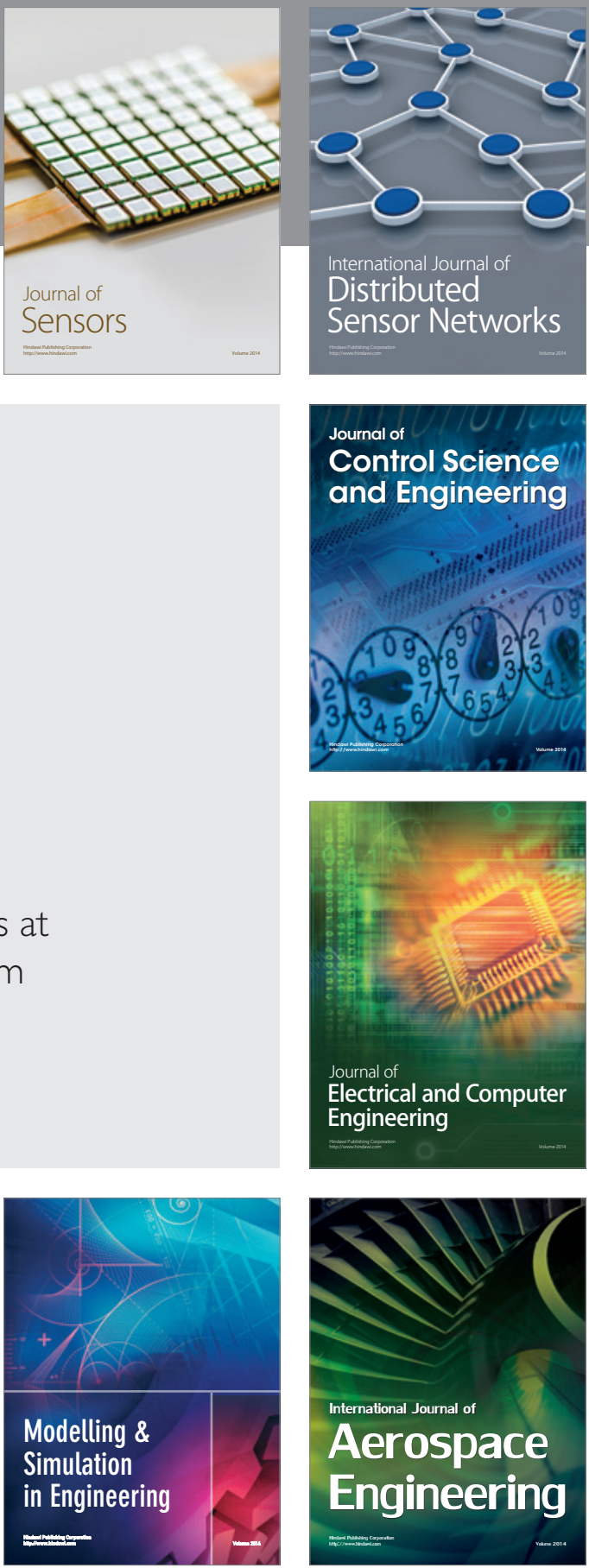

Journal of

Control Science

and Engineering
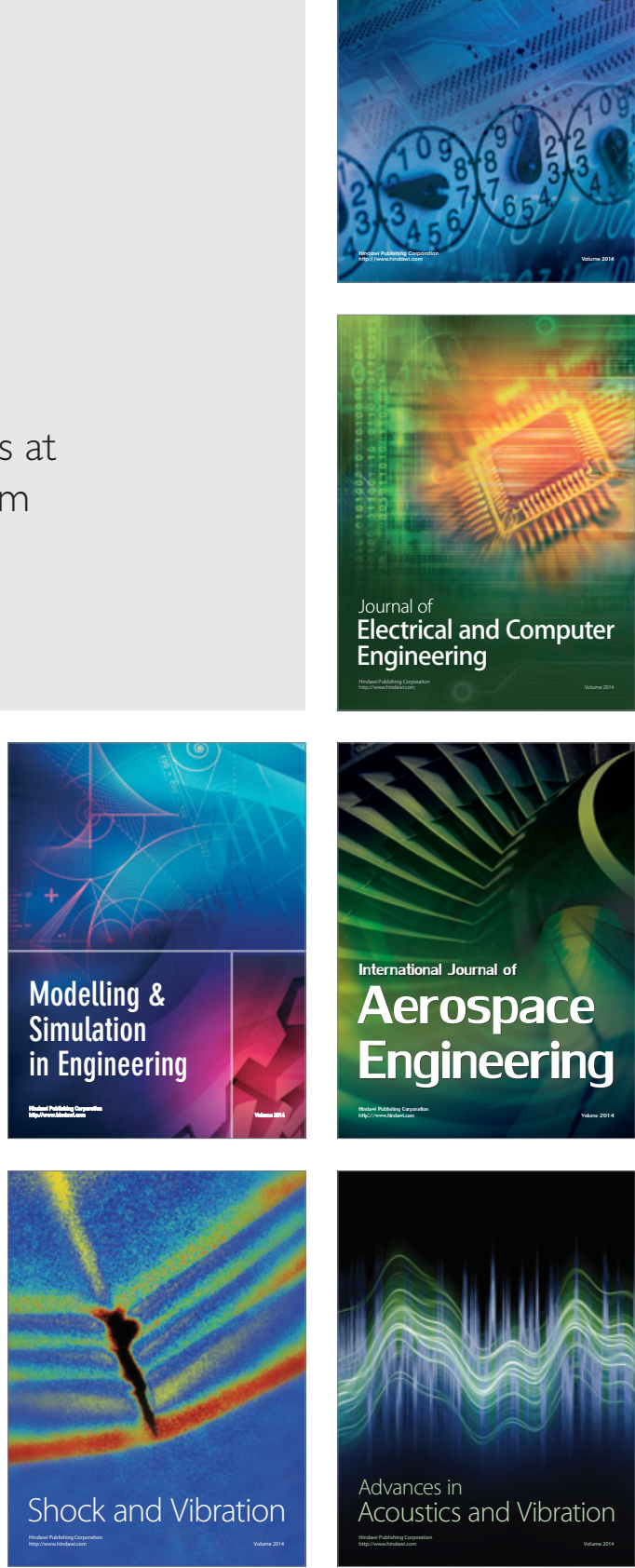